\title{
Approximate *-derivations and approximate quadratic *-derivations on $C^{*}$-algebras
}

\author{
Sun Young Jang ${ }^{1}$ and Choonkil Park ${ }^{2^{*}}$
}

\author{
* Correspondence: baak@hanyang. \\ ac.kr \\ 2Department of Mathematics, \\ Research Institute for Natural \\ Sciences, Hanyang University, Seoul \\ 133-791, Korea \\ Full list of author information is \\ available at the end of the article
}

\begin{abstract}
In this paper, we prove the stability of *-derivations and of quadratic *-derivations on Banach *-algebras. We moreover prove the superstability of ${ }^{*}$-derivations and of quadratic *-derivations on $C^{*}$-algebras.

2000 Mathematics Subject Classification: 39B52; 47B47; 46L05; $39 B 72$.
\end{abstract}

Keywords: *-derivation, quadratic *-derivation, $C^{*}$-algebra; stability, superstability

\section{Introduction and preliminaries}

Suppose that $\mathcal{A}$ is a complex Banach *-algebra. A $\mathbb{C}$-linear mapping $\delta: D(\delta) \rightarrow \mathcal{A}$ is said to be a derivation on $\mathcal{A}$ if $\delta(a b)=\delta(a)+b+a \delta(b)$ for all $a, b \in \mathcal{A}$, where $D(\delta)$ is a domain of $\delta$ and $D(\delta)$ is dense in $\mathcal{A}$. If $\delta$ satisfies the additional condition $\delta\left(a^{*}\right)=\delta(a)^{*}$ for all $a \in \mathcal{A}$, then $\delta$ is called a ${ }^{*}$-derivation on $\mathcal{A}$. It is well known that if $\mathcal{A}$ is a $C^{*}$ algebra and $D(\delta)$ is $\mathcal{A}$, then the derivation $\delta$ is bounded.

A $C^{*}$-dynamical system is a triple $(\mathcal{A}, \mathrm{G}, \alpha)$ consisting of a $C^{*}$-algebra $\mathcal{A}$, a locally compact group $G$, and a pointwise norm continuous homomorphism $\alpha$ of $G$ into the group $\operatorname{Aut}(\mathcal{A})$ of *-automorphisms of $\mathcal{A}$. Every bounded *-derivation $\delta$ arises as an infinitesimal generator of a dynamical system for $\mathbb{R}$. In fact, if $\delta$ is a bounded *-derivation of $\mathcal{A}$ on a Hilbert space $\mathcal{H}$, then there exists an element $h$ in the enveloping von Neumann algebra $\mathcal{A}^{\prime \prime}$ such that

$$
\delta(x)=\operatorname{ad}_{i h}(x)
$$

for all $x \in \mathcal{A}$.

If, for each $t \in \mathbb{R}, \alpha_{t}$ is defined by $\alpha_{t}(x)=e^{i}$ th $x e^{-i}$ th for all $x \in \mathcal{A}$, then $\alpha_{t}$ is a *-automorphism of $\mathcal{A}$ induced by unitaries $U_{t}=e^{i}$ th for each $t \in \mathbb{R}$. The action $\alpha: \mathbb{R} \rightarrow \operatorname{Aut}(\mathcal{A}), t \rightarrow \alpha_{t}$, is a strongly continuous one-parameter group of *automorphisms of $\mathcal{A}$. For several reasons, the theory of bounded derivations of $C^{*}$-algebras is important in the quantumn mechanics (see [1-3]).

A functional equation is called stable if any function satisfying the functional equation "approximately" is near to a true solution of the functional equation. We say that a functional equation is superstable if every approximate solution is an exact solution of it (see [4]).

In 1940, Ulam [5] proposed the following question concerning stability of group homomorphisms: under what condition does there exist an additive mapping near an approximately additive mapping? Hyers [6] answered the problem of Ulam for the 
case where $G_{1}$ and $G_{2}$ are Banach spaces. A generalized version of the theorem of Hyers for an approximately linear mapping was given by Rassias [7]. Since then, the stability problems of various functional equations have been extensively investigated by a number of authors (see [8-19]). In particular, those of the important functional equations are the following functional equations

$$
\begin{aligned}
& f(x+y)=f(x)+f(y), \\
& 2 f\left(\frac{x+y}{2}\right)=f(x)+f(y),
\end{aligned}
$$

which are called the Cauchy functional equation and the Jensen functional equation, respectively. The function $f(x)=b x$ is a solution of these functional equations. Every solution of the functional equations (1.1) and (1.2) is said to be an additive mapping.

In this paper, we introduce functional equations of *-derivations and of quadratic "-derivations. we prove the stability of "-derivations associated with the Cauchy functional equation and the Jensen functional equation and of quadratic "-derivations on Banach "-algebra. We moreover prove the superstability of "-derivations and of quadratic "-derivations on $C^{*}$-algebras.

\section{Stability of *-derivations on Banach *-algebras}

In this section, let $\mathcal{A}$ be a Banach "-algebra. We prove the stability of *-derivations on $\mathcal{A}$.

Theorem 2.1 Suppose that $f: \mathcal{A} \rightarrow$ Ais a mapping with $f(0)=0$ for which there exists a function $\varphi: \mathcal{A}^{4} \rightarrow[0, \infty)$ such that

$$
\begin{aligned}
& \tilde{\varphi}(a, b, c, d):=\sum_{n=0}^{\infty} \frac{1}{2^{n+1}} \varphi\left(2^{n} a, 2^{n} b, 2^{n} c, 2^{n} d\right)<\infty, \\
& \|f(\lambda a+b+c d)-\lambda f(a)-f(b)-f(c) d-c f(d)\| \leq \varphi(a, b, c, d), \\
& \left\|f\left(a^{*}\right)-f(a)^{*}\right\| \leq \varphi(a, a, a, a)
\end{aligned}
$$

for all $\lambda \in \mathbb{T}:=\{\lambda \in \mathbb{C}:|\lambda|=1\}$ and all $a, b, c, d \in \mathcal{A}$. Then there exists a unique *-derivation $\delta$ on $\mathcal{A}$ satisfying

$$
\|f(a)-\delta(a)\| \leq \tilde{\varphi}(a, a, 0,0),
$$

for all $a \in \mathcal{A}$.

Proof. Setting $a=b, c=d=0$ and $\lambda=1$ in (2.2), we have

$$
\|f(2 a)-2 f(a)\| \leq \varphi(a, a, 0,0)
$$

for all $a \in \mathcal{A}$. One can use induction to show that

$$
\left\|\frac{f\left(2^{n} a\right)}{2^{n}}-\frac{f\left(2^{m} a\right)}{2^{m}}\right\|=\sum_{k=m}^{n-1} \frac{1}{2^{k+1}} \varphi\left(2^{k} a, 2^{k} a, 0,0\right)
$$

for all $n>m \geq 0$ and all $a \in \mathcal{A}$. It follows from (2.5) and (2.1) that the sequence $\left\{\frac{f\left(2^{n} a\right)}{2^{n}}\right\}$ is Cauchy. Due to the completeness of $\mathcal{A}$, this sequence is convergent. Define 


$$
\delta(a):=\lim _{n \rightarrow \infty} \frac{f\left(2^{n} a\right)}{2^{n}}
$$

for all $a \in \mathcal{A}$. Then, we have

$$
\delta\left(\frac{1}{2^{k}} a\right)=\lim _{n \rightarrow \infty} \frac{1}{2^{k}} \frac{f\left(2^{n-k} a\right)}{2^{n-k}}=\frac{1}{2^{k}} \delta(a)
$$

for each $k \in \mathbb{N}$. Putting $c=d=0$ and replacing $a$ and $b$ by $2^{n} a$ and $2^{n} b$, respectively, in (2.2), we get

$$
\left\|\frac{1}{2^{n}} f\left(2^{n}(\lambda a+b)\right)-\lambda \frac{1}{2^{n}} f\left(2^{n} a\right)-\frac{1}{2^{n}} f\left(2^{n} b\right)\right\| \leq \frac{1}{2^{n}} \varphi\left(2^{n} a, 2^{n} b, 0,0\right) .
$$

Taking the limit as $n \rightarrow \infty$, we obtain

$$
\delta(\lambda a+b)=\lambda \delta(a)+\delta(b)
$$

for all $a, b \in \mathcal{A}$ and all $\lambda \in \mathbb{T}$. Putting $a=b=0$ and replacing $c$ and $d$ by $2^{n} c$ and $2^{n} d$, respectively, in (2.2), we get

$$
\begin{array}{r}
\left\|\frac{1}{2^{2 n}} f\left(2^{2 n} c d\right)-\frac{1}{2^{2 n}} f\left(2^{n} c\right)\left(2^{n} d\right)-\frac{1}{2^{2 n}}\left(2^{n} c\right) f\left(2^{n} d\right)\right\| \\
\leq \frac{1}{2^{2 n}} \varphi\left(0,0,2^{n} c, 2^{n} d\right) \leq \frac{1}{2^{n}} \varphi\left(0,0,2^{n} c, 2^{n} d\right) .
\end{array}
$$

Taking the limit as $n \rightarrow \infty$, we obtain

$$
\delta(c d)=\delta(c) d+c \delta(d)
$$

for all $c, d \in \mathcal{A}$.

Next, let $\lambda=\lambda_{1}+\mathrm{i} \lambda_{2} \in \mathbb{C}$ where $\lambda_{1}, \lambda_{2}, \in \mathbb{R}$. Let $\gamma_{1}=\lambda_{1}-\left[\lambda_{1}\right]$ and $\gamma_{2}=\lambda_{2}-\left[\lambda_{2}\right]$, where $[\lambda]$ denotes the integer part of $\lambda$. Then, $0 \leq \gamma_{1}<1(1 \leq i \leq 2)$. One can represent $\gamma_{i}$ as $\gamma_{i}=\frac{\lambda_{i, 1}+\lambda_{i, 2}}{2}$ such that $\lambda_{i, j} \in \mathbb{T}(1 \leq i, j \leq 2)$. From (2.7) and (2.8), it follows that

$$
\begin{aligned}
\delta(\lambda a) & =\delta\left(\lambda_{1} a\right)+\mathrm{i} \delta\left(\lambda_{2} a\right) \\
& =\left(\left[\lambda_{1}\right] \delta(a)+\delta\left(\gamma_{1} a\right)\right)+\mathrm{i}\left(\left[\lambda_{2}\right] \delta(a)+\delta\left(\gamma_{2} a\right)\right) \\
& =\left(\left[\lambda_{1}\right] \delta(a)+\frac{1}{2} \delta\left(\lambda_{1,1} a+\lambda_{1,2} a\right)\right)+\mathrm{i}\left(\left[\lambda_{2}\right] \delta(a)+\frac{1}{2} \delta\left(\lambda_{2,1} a+\lambda_{2,2} a\right)\right) \\
& =\left(\left[\lambda_{1}\right] \delta(a)+\frac{1}{2} \lambda_{1,1} \delta(a)+\frac{1}{2} \lambda_{1,2} \delta(a)\right)+\mathrm{i}\left(\left[\lambda_{2}\right] \delta(a)+\frac{1}{2} \lambda_{2,1} \delta(a)+\frac{1}{2} \lambda_{2,2} \delta(a)\right) \\
& =\lambda_{1} \delta(a)+\mathrm{i} \lambda_{2} \delta(a)=\lambda \delta(a)
\end{aligned}
$$

for all $a \in \mathcal{A}$. Hence, $\delta$ is $\mathbb{C}$-linear, and so it is a derivation on $\mathcal{A}$. Moreover, it follows from (2.5) with $m=0$ and (2.6) that $\|\delta(a)-f(a)\| \leq \tilde{\varphi}(a, a, 0,0)$ for all $a \in \mathcal{A}$. It is well known that the additive mapping $\delta$ satisfying (2.4) is unique (see [3] or [19]). Replacing $a$ and $a^{*}$ by $2^{n} a$ and $2^{n} a^{*}$, respectively, in (2.3), we get

$$
\left\|\frac{1}{2^{n}} f\left(2^{n} a^{*}\right)-\frac{1}{2^{n}} f\left(2^{n} a\right)^{*}\right\| \leq \frac{1}{2^{n}} \varphi\left(2^{n} a, 2^{n} a, 2^{n} a, 2^{n} a\right) .
$$

Passing to the limit as $n \rightarrow \infty$, we get the $\delta\left(a^{*}\right)=\delta(a)^{* *}$ for all $a \in \mathcal{A}$. So $\delta$ is a *-derivation on $\mathcal{A}$, as desired. $\square$

Corollary 2.2 Let $\varepsilon, p$ be positive real numbers with $p<1$. Suppose that $f: \mathcal{A} \rightarrow \mathcal{A}$ is a mapping satisfying 


$$
\begin{aligned}
& \|f(\lambda a+b+c d)-\lambda f(a)-f(b)-c f(d)-f(c) d\| \leq \varepsilon\left(\|a\|^{p}+\|b\|^{p}+\|c\|^{p}+\|d\|^{p}\right), \\
& \left\|f\left(a^{*}\right)-f(a)^{*}\right\| \leq 4 \varepsilon\|a\|^{p}
\end{aligned}
$$

for all $\lambda \in \mathbb{T}$ and all $a, b, c, d \in \mathcal{A}$. Then there exists a unique "-derivation $\delta$ on $\|f(a)-\delta(a)\| \leq \frac{2 \varepsilon}{2-2^{p}}\|a\|^{p_{\text {satisfying }}}$

$$
\|f(a)-\delta(a)\| \leq \frac{2 \varepsilon}{2-2^{p}}\|a\|^{p}
$$

for all $a \in \mathcal{A}$.

Proof. Putting $\phi(a, b, c, d)=\varepsilon\left(\|a\|^{p}+\|b\|^{p}+\|c\|^{p}+\|\left. d\right|^{p}\right)$ in Theorem 2.1, we get the desired result.

Similarly, we can obtain the following. We will omit the proof.

Theorem 2.3 Suppose that $f: \mathcal{A} \rightarrow \mathcal{A}$ is a mapping with $f(0)=0$ for which there exists a function $\varphi: \mathcal{A}^{4} \rightarrow[0, \infty)$ satisfying (2.2), (2.3) and

$$
\sum_{n=1}^{\infty} 2^{2 n-1} \varphi\left(\frac{a}{2^{n}}, \frac{b}{2^{n}}, \frac{c}{2^{n}}, \frac{d}{2^{n}}\right)<\infty
$$

for all $a, b, c, d \in \mathcal{A}$. Then there exists a unique "-derivation $\delta$ on Asatisfying

$$
\|f(a)-\delta(a)\| \leq \tilde{\varphi}(a, a, 0,0),
$$

for all $a \in \mathcal{A}$, where

$$
\tilde{\varphi}(a, b, c, d):=\sum_{n=1}^{\infty} 2^{n-1} \varphi\left(\frac{a}{2^{n}}, \frac{b}{2^{n}}, \frac{c}{2^{n}}, \frac{d}{2^{n}}\right) .
$$

Corollary 2.4 Let $\varepsilon, p$ be positive real numbers with $p>2$. Suppose that $f: \mathcal{A} \rightarrow \mathcal{A}$ is a mapping satisfying (2.10) and (2.11). Then there exists a unique "-derivation $\delta$ on $\|f(a)-\delta(a)\| \leq \frac{2 \varepsilon}{2^{p}-2}\|a\|^{p_{\text {satisfying }}}$

$$
\|f(a)-\delta(a)\| \leq \frac{2 \varepsilon}{2^{p}-2}\|a\|^{p}
$$

for all $a \in \mathcal{A}$.

Proof. Putting $\phi(a, b, c, d)=\varepsilon\left(\|a\|^{p}+\|b\|^{p}+\|c\|^{p}+\|\left. d\right|^{p}\right)$ in Theorem 2.3, we get the desired result. $\square$

\section{Stability of *-derivations associated with the Jensen functional equation}

The stability of the Jensen functional equation has been studied first by Kominek and then by several other mathematicians (see $[11,20]$ ).

In this section, we study the stability of "-derivation associated with the Jensen functional equation in a Banach "-algebra $\mathcal{A}$.

Theorem 3.1 Let $\mathcal{A}$ be a Banach"-algebra. Suppose that $f: \mathcal{A} \rightarrow \mathcal{A}$ is a mapping with $f(0)=0$ for which there exists a function $\varphi: \mathcal{A} \times \mathcal{A} \rightarrow[0, \infty)$ such that

$$
\widetilde{\varphi}(a, b):=\sum_{n=0}^{\infty} \frac{1}{3^{n}} \varphi\left(3^{n} a, 3^{n} b\right)<\infty,
$$




$$
\begin{aligned}
& \left\|2 f\left(\frac{\lambda a+\lambda b}{2}\right)-\lambda f(a)-\lambda f(b)\right\| \leq \varphi(a, b), \\
& \left\|f\left(a^{*}\right)-f(a)^{*}\right\| \leq \varphi(a, a) \\
& \|f(a b)-a f(b)-f(a) b\| \leq \varphi(a, b)
\end{aligned}
$$

for all $a, b \in \mathcal{A}$ and all $\lambda \in \mathbb{T}$. Then there exists a unique *-derivation $\delta$ on $\mathcal{A}$ satisfying

$$
\|f(a)-\delta(a)\| \leq \frac{1}{3}(\tilde{\varphi}(a,-a)+\tilde{\varphi}(-a, 3 a))
$$

for all $a \in \mathcal{A}$.

Proof. Letting $\lambda=1$ and $b=-a$ in (3.2), we get

$$
\|-f(a)-f(-a)\| \leq \varphi(a,-a)
$$

for all $a \in \mathcal{A}$. Letting $\lambda=1$ and replacing $a$ and $b$ by $-a$ and $3 a$, respectively, in (3.2), we get

$$
\|2 f(a)-f(-a)-f(3 a)\| \leq \varphi(-a, 3 a)
$$

for all $a \in \mathcal{A}$. Thus,

$$
\begin{aligned}
\left\|f(a)-\frac{1}{3} f(3 a)\right\| & \leq \frac{1}{3}(\|f(a)+f(-a)\|+\|2 f(a)-f(-a)-f(3 a)\|) \\
& \leq \frac{1}{3}(\varphi(a,-a)+\varphi(-a, 3 a))
\end{aligned}
$$

for all $a \in \mathcal{A}$. So

$$
\begin{aligned}
\left\|\frac{1}{3^{n}} f\left(3^{n} a\right)-\frac{1}{3^{m}} f\left(3^{m} a\right)\right\| & \leq \sum_{j=m}^{n-1}\left\|\frac{1}{3^{j}} f\left(3^{j} a\right)-\frac{1}{3^{j+1}} f\left(3^{j+1} a\right)\right\| \\
& \leq \frac{1}{3} \sum_{j=m}^{n-1} \frac{1}{3^{j}}\left(\varphi\left(3^{j} a,-3^{j} a\right)+\varphi\left(-3^{j} a, 3^{j+1} a\right)\right)
\end{aligned}
$$

for all nonnegative integers $n, m$ with $n>m$ and all $a \in \mathcal{A}$. It follows from (3.6) that the sequence $\left\{\frac{1}{3^{n}} f\left(3^{n} a\right)\right\}$ is a Cauchy sequence for all $a \in \mathcal{A}$. Since $\mathcal{A}$ is complete, the sequence $\left\{\frac{1}{3^{n}} f\left(3^{n} a\right)\right\}$ is convergent. So one can define the mapping $\delta: \mathcal{A} \rightarrow \mathcal{A}$ by

$$
\delta(a)=\lim _{n \rightarrow \infty} \frac{1}{3^{n}} f\left(3^{n} a\right)
$$

for all $a \in \mathcal{A}$. By (3.2),

$$
\begin{aligned}
\left\|2 \delta\left(\frac{a+b}{2}\right)-\delta(a)-\delta(b)\right\| & =\lim _{n \rightarrow \infty} \frac{1}{3^{n}}\left\|2 f\left(3^{n} \frac{a+b}{2}\right)-f\left(3^{n} a\right)-f\left(3^{n} b\right)\right\| \\
& \leq \lim _{n \rightarrow \infty} \frac{1}{3^{n}} \varphi\left(3^{n} a, 3^{n} b\right)=0
\end{aligned}
$$

for all $a, b \in \mathcal{A}$. Thus

$$
2 \delta\left(\frac{a+b}{2}\right)=\delta(a)+\delta(b)
$$


for all $a, b \in \mathcal{A}$. Since $f(0)=0$, we have $\delta(0)=0$. Putting $b=0$ in (3.7), we get $2 \delta\left(\frac{a}{2}\right)=\delta(a)$ for all $a \in \mathcal{A}$ and therefore $\delta(a)+\delta(b)=2 \delta\left(\frac{a+b}{2}\right)=\delta(a+b)$ for all $a, b \in \mathcal{A}$. Moreover, letting $m=0$ and passing the limit $n \rightarrow \infty$ in (3.6), we get (3.5).

Replacing both $a$ and $b$ in (3.2) by $3^{n} a$ and then dividing both sides of the obtained inequality by $3^{n}$, we get

$$
\left\|\frac{1}{3^{n}} f\left(\lambda 3^{n} a\right)-\frac{\lambda}{3^{n}} f\left(3^{n} a\right)\right\| \leq \frac{1}{3^{n}} \varphi\left(3^{n} a, 3^{n} a\right) .
$$

Passing the limit as $n \rightarrow \infty$, we get $\delta(\lambda a)=\lambda \delta(a)$ for all $\lambda \in \mathbb{T}$. Thus we can get $\delta(\lambda a)$ $=\lambda \delta(a)$ for all $\lambda \in \mathbb{C}$ by the similar discussion in the proof of Theorem 2.1.

Replacing $a$ in (3.3) by $3^{n} a$ and then dividing the both sides of the obtained inequality by $3^{\text {n }}$, we get

$$
\left\|\frac{1}{3^{n}} f\left(3^{n} a^{*}\right)-\frac{1}{3^{n}} f\left(3^{n} a\right)^{*}\right\| \leq \frac{1}{3^{n}} \varphi\left(3^{n} a, 3^{n} a\right) .
$$

Passing the limit as $n$ tends to infinity, we get $\delta\left(a^{*}\right)=\delta(a)^{*}$.

Similarly, replacing $a$ and $b$ in (3.4) by $3^{n} a$ and $3^{n} b$, respectively, we get

$$
\left\|\frac{f\left(3^{2 n} a b\right)}{3^{2 n}}-\frac{3^{n} a f\left(3^{n} b\right)}{3^{2 n}}-\frac{f\left(3^{n} a\right)\left(3^{n} b\right)}{3^{2 n}}\right\| \leq \frac{1}{3^{2 n}} \varphi\left(3^{n} a, 3^{n} b\right) \leq \frac{1}{3^{n}} \varphi\left(3^{n} a, 3^{n} b\right),
$$

which tends to zero, as $n$ tends to $\infty$. So we get $\delta(a b)=\delta(a) d+a \delta(b)$ for all $a, b \in \mathcal{A}$. Hence, $\delta$ is a ${ }^{*}$-derivation on $\mathcal{A}$.

Corollary 3.2 Let $\varepsilon$, $p$ be positive real numbers with $p<1$. Suppose that $f: \mathcal{A} \rightarrow \mathcal{A}$ is a mapping satisfying

$$
\begin{aligned}
& \left\|2 f\left(\frac{\lambda a+\lambda b}{2}\right)-\lambda f(a)-\lambda f(b)\right\| \leq \varepsilon\left(\|a\|^{p}+\|b\|^{p}\right), \\
& \left\|f\left(a^{*}\right)-f(a)^{*}\right\| \leq 2 \varepsilon\|a\|^{p}, \\
& \|f(a b)-a f(b)-f(a) b\| \leq \varepsilon\left(\|a\|^{p}+\|b\|^{p}\right)
\end{aligned}
$$

for all $\lambda \in \mathbb{T}$ and all $a, b \in \mathcal{A}$. Then there exists a unique ${ }^{*}$-derivation $\delta$ on $\mathcal{A}$ satisfying

$$
\|f(a)-\delta(a)\| \leq \frac{3+3^{p}}{3-3^{p}} \varepsilon\|a\|^{p}
$$

for all $a \in \mathcal{A}$.

Proof. Putting $\phi(a, b)=\varepsilon\left(\|a\|^{p}+\|b\|^{p}\right)$ in Theorem 3.1, we get the desired result. $\square$ Similarly, we can obtain the following. We will omit the proof.

Theorem 3.3 Let $\mathcal{A}$ be a Banach "-algebra. Suppose that $f: \mathcal{A} \rightarrow \mathcal{A}$ is a mapping with $f(0)=0$ for which there exists a function $\|f(a)-\delta(a)\| \leq \frac{2 \varepsilon}{2^{p}-2} \|$ a $\|^{p_{\text {satisfying }}}$ (3.2), (3.3), (3.4) and

$$
\sum_{n=1}^{\infty} 3^{2 n} \varphi\left(\frac{a}{3^{n}}, \frac{b}{3^{n}}\right)<\infty
$$


for all $a, b \in \mathcal{A}$. Then there exists a unique *-derivation $\delta$ on $\mathcal{A}$ satisfying

$$
\|f(a)-\delta(a)\| \leq \frac{1}{3}(\tilde{\varphi}(a,-a)+\tilde{\varphi}(-a, 3 a))
$$

for all $a \in \mathcal{A}$, where

$$
\widetilde{\varphi}(a, b):=\sum_{n=1}^{\infty} 3^{n} \varphi\left(\frac{a}{3^{n}}, \frac{b}{3^{n}}\right) .
$$

Corollary 3.4 Let $\varepsilon, p$ be positive real numbers with $p>2$. Suppose that $f: \mathcal{A} \rightarrow \mathcal{A}$ is a mapping satisfying (3.8), (3.9) and (3.10). Then there exists a unique "-derivation $\delta$ on Asatisfying

$$
\|f(a)-\delta(a)\| \leq \frac{3^{p}+3}{3^{p}-3} \varepsilon\|a\|^{p}
$$

for all $a \in \mathcal{A}$.

Proof. Putting $\phi(a, b)=\varepsilon\left(\|a\|^{p}+\|b\|^{p}\right)$ in Theorem 3.3, we get the desired result. $\square$

\section{Stability of quadratic *-derivations on Banach *-algebras}

In this section, we prove the stability of quadratic *-derivations on a Banach *-algebra $\mathcal{A}$.

Definition 4.1 Let $\mathcal{A}$ be $a^{*}$-normed algebra. A mapping $\delta: \mathcal{A} \rightarrow \mathcal{A}$ is a quadratic "-derivation on $\mathcal{A}$ if $\delta$ satisfies the following properties:

(1) $\delta$ is a quadratic mapping,

(2) $\delta$ is quadratic homogeneous, that is, $\delta(\lambda a)=\lambda^{2} \delta(a)$ for all $a \in \mathcal{A}$ and all $\lambda \in \mathbb{C}$,

(3) $\delta(a b)=\delta(a) b^{2}+a^{2} \delta(b)$ for all $a, b \in \mathcal{A}$,

(4) $\delta\left(a^{*}\right)=\delta(a)^{*}$ for all $a \in \mathcal{A}$.

Theorem 4.2 Suppose that $f: \mathcal{A} \rightarrow$ Ais a mapping with $f(0)=0$ for which there exists a function $\varphi: \mathcal{A}^{4} \rightarrow[0, \infty)$ such that

$$
\begin{aligned}
& \tilde{\varphi}(a, b, c, d):=\sum_{k=0}^{\infty} \frac{1}{4^{k}} \varphi\left(2^{k} a, 2^{k} b, 2^{k} c, 2^{k} d\right)<\infty, \\
& \| f(\lambda a+\lambda b+c d)+f(\lambda a-\lambda b+c d)-2 \lambda^{2} f(a)-2 \lambda^{2} f(b)-2 f(c) d^{2}-2 c^{2} f(d) \\
& \quad \leq \varphi(a, b, c, d), \\
& \left\|f\left(a^{*}\right)-f(a)^{*}\right\| \leq \varphi(a, a, a, a)
\end{aligned}
$$

for all $a, b, c, d \in$ Aand all $\lambda \in \mathbb{T}$. Also, if for each fixed $a \in \mathcal{A}$ the mapping $t \rightarrow f(t a)$ from $\mathbb{R}$ to $\mathcal{A}$ is continuous, then there exists a unique quadratic "-derivation $\delta$ on $\|f(a)-\delta(a)\| \leq \frac{1}{4} \tilde{\varphi}(a, a, 0,0)$ satisfying

$$
\|f(a)-\delta(a)\| \leq \frac{1}{4} \tilde{\varphi}(a, a, 0,0)
$$

for all $a \in \mathcal{A}$. 
Proof. Putting $a=b, c=d=0$, , and $\lambda=1$ in (4.1), we have

$$
\|f(2 a)-4 f(a)\| \leq \varphi(a, a, 0,0)
$$

for all $a \in \mathcal{A}$. One can use induction to show that

$$
\left\|\frac{f\left(2^{n} a\right)}{4^{n}}-\frac{f\left(2^{m} a\right)}{4^{m}}\right\| \leq \frac{1}{4} \sum_{k=m}^{n-1} \frac{\varphi\left(2^{k} a, 2^{k} a, 0,0\right)}{4^{k}}
$$

for all $n>m \geq 0$ and all $a \in \mathcal{A}$. It follows from (4.3) that the sequence $\left\{\frac{f\left(2^{n} a\right)}{4^{n}}\right\}$ is Cauchy. Since $\mathcal{A}$ is complete, this sequence is convergent. Define

$$
\delta(a):=\lim _{n \rightarrow \infty} \frac{f\left(2^{n} a\right)}{4^{n}} .
$$

Since $f(0)=0$, we have $\delta(0)=0$. Replacing $a$ and $b$ by $2^{n} a$ and $2^{n} b, c=d=0$, respectively, in (4.1), we get

$$
\left\|\frac{f\left(2^{n}(\lambda a+\lambda b)\right)}{4^{n}}+\frac{f\left(2^{n}(\lambda a-\lambda b)\right)}{4^{n}}-2 \lambda^{2} \frac{f\left(2^{n} a\right)}{4^{n}}-2 \lambda^{2} \frac{f\left(2^{n} b\right)}{4^{n}}\right\| \leq \frac{\varphi\left(2^{n} a, 2^{n} b, 0,0\right)}{4^{n}} .
$$

Taking the limit as $n \rightarrow \infty$, we obtain

$$
\delta(\lambda a+\lambda b)+\delta(\lambda a-\lambda b)=2 \lambda^{2} \delta(a)+2 \lambda^{2} \delta(b)
$$

for all $a, b \in \mathcal{A}$ and all $\lambda \in \mathbb{T}$. Putting $\lambda=1$ in (4.4), we obtain that $\delta$ is a quadratic mapping. Setting $b:=a$ in (4.4), we get

$$
\delta(2 \lambda a)=4 \lambda^{2} \delta(a)
$$

for all $a \in \mathcal{A}$ and all $\lambda \in \mathbb{T}$. Hence,

$$
\delta(\lambda a)=\lambda^{2} \delta(a)
$$

for all $a \in \mathcal{A}$ and all $\lambda \in \mathbb{T}$. Under the assumption that $f(t a)$ is continuous in $t \in \mathbb{R}$ for each fixed $a \in \mathcal{A}$, by the same reasoning as in the proof of [10], we obtain that $\delta(\lambda a)=\lambda^{2} \delta(a)$ for all $a \in \mathcal{A}$ and all $\lambda \in \mathbb{R}$. Hence,

$$
\delta(\lambda a)=\delta\left(\frac{\lambda}{|\lambda|}|\lambda| a\right)=\frac{\lambda^{2}}{|\lambda|^{2}} \delta(|\lambda| a)=\frac{\lambda^{2}}{|\lambda|^{2}}|\lambda|^{2} \delta(a)=\lambda^{2} \delta(a)
$$

for all $a \in \mathcal{A}$ and all $\lambda \in \mathbb{C}(\lambda \neq 0)$. This means that $\delta$ is quadratic homogeneous.

Replacing $c$ and $d$ by $2^{n} c$ and $2^{n} d$, respectively, and putting $a=b=0$ in (4.1), we get

$$
\begin{aligned}
& \left\|\frac{f\left(2^{n} c \cdot 2^{n} d\right)}{4^{2 n}}+\frac{f\left(2^{n} c \cdot 2^{n} d\right)}{4^{2 n}}-2 \frac{2^{2 n} c^{2} f\left(2^{n} d\right)}{4^{2 n}}-2 \frac{f\left(2^{n} c\right) 2^{2 n} d^{2}}{4^{2 n}}\right\| \\
& \quad=\left\|\frac{f\left(2^{2 n} c d\right)}{4^{2 n}}+\frac{f\left(2^{2 n} c d\right)}{4^{2 n}}-2 \frac{2^{2 n} c^{2}}{2^{2 n}} \frac{f\left(2^{n} d\right)}{4^{n}}-2 \frac{f\left(2^{n} c\right)}{4^{n}} \frac{2^{2 n} d^{2}}{2^{2 n}}\right\| \\
& \quad \leq \frac{\varphi\left(0,0,2^{n} c, 2^{n} d\right)}{4^{2 n}} \leq \frac{\varphi\left(0,0,2^{n} c, 2^{n} d\right)}{4^{n}}
\end{aligned}
$$

for all $c, d \in \mathcal{A}$. 
Hence, we have

$$
\left\|\delta(c d)-c^{2} \delta(d)-\delta(c) d^{2}\right\| \leq \lim _{n \rightarrow \infty} \frac{\varphi\left(0,0,2^{n} c, 2^{n} d\right)}{4^{n}}=0 .
$$

Thus, $\delta$ is a quadratic "-derivation on $\mathcal{A}$.

The rest of the proof is similar to the proof of Theorem 2.1.

Corollary 4.3 Let $\varepsilon, p$ be positive real numbers with $p<2$. Suppose that $f: \mathcal{A} \rightarrow \mathcal{A}$ is a mapping such that

$$
\begin{aligned}
& \left\|f(\lambda a+\lambda b+c d)+f(\lambda a-\lambda b+c d)-2 \lambda^{2} f(a)-2 \lambda^{2} f(b)-2 c^{2} f(d)-2 f(c) d^{2}\right\| \\
& \quad \leq \varepsilon\left(\|a\|^{p}+\|b\|^{p}+\|c\|^{p}+\|d\|^{p}\right)
\end{aligned}
$$

for all $a, b, c, d \in \mathcal{A}$ and all $\lambda \in \mathbb{T}$. Also, if for each fixed $a \in \mathcal{A}$ the mapping $t \rightarrow f(t a)$ is continuous, then there exists a unique derivation $\delta$ on Asatisfying

$$
\|f(a)-\delta(a)\| \leq \frac{2 \varepsilon}{4-2^{p}}\|a\|^{p}
$$

for all $a \in \mathcal{A}$.

Proof. Putting $\phi(a, b, c, d)=\varepsilon\left(\|a\|^{p}+\|b\|^{p}+\|c\|^{p}+\|d\|^{p}\right)$ in Theorem 4.2, we get the desired result.

Similarly, we can obtain the following. We will omit the proof.

Theorem 4.4 Suppose that $f: \mathcal{A} \rightarrow \mathcal{A}$ is a mapping with $f(0)=0$ for which there exists a function $\varphi: \mathcal{A}^{4} \rightarrow[0, \infty)$ satisfying (4.1), (4.2) and

$$
\sum_{k=1}^{\infty} 4^{2 k} \varphi\left(\frac{a}{2^{k}}, \frac{b}{2^{k}}, \frac{c}{2^{k}}, \frac{d}{2^{k}}\right)<\infty
$$

for all $a, b, c, d \in \mathcal{A}$. Also, if for each fixed $a \in \mathcal{A}$ the mapping $t \rightarrow f(t a)$ from $\mathbb{R}$ to $\mathcal{A}$ is continuous, then there exists a unique quadratic "-derivation $\delta$ on $\mathcal{A}$ satisfying

$$
\|f(a)-\delta(a)\| \leq \frac{1}{4} \tilde{\varphi}(a, a, 0,0)
$$

for all $a \in \mathcal{A}$, where

$$
\tilde{\varphi}(a, b, c, d):=\sum_{k=1}^{\infty} 4^{k} \varphi\left(\frac{a}{2^{k^{\prime}}}, \frac{b}{2^{k}}, \frac{c}{2^{k}}, \frac{d}{2^{k}}\right)
$$

Corollary 4.5 Let $\varepsilon, p$ be positive real numbers with $p>4$. Suppose that $f: \mathcal{A} \rightarrow \mathcal{A}$ is a mapping satisfying (4.5). Also, if for each fixed $a \in \mathcal{A}$ the mapping $t \rightarrow f(t a)$ is continuous, then there exists a unique derivation $\delta$ on Asatisfying

$$
\|f(a)-\delta(a)\| \leq \frac{2 \varepsilon}{2^{p}-4}\|a\|^{p}
$$

for all $a \in \mathcal{A}$.

Proof. Putting $\phi(a, b, c, d)=\varepsilon\left(\|a\|^{p}+\|b\|^{p}+\|c\|^{p}+\|d\|^{p}\right)$ in Theorem 4.4, we get the desired result. 


\section{Superstability of *-derivations and of quadratic *-derivations On $C^{*}$ - algebras}

We prove the superstability of "-derivations and of quadratic "-derivations on $C^{*}$-algebras. More precisely, we introduce the concept of $(\psi, \varepsilon)$-approximate "-derivations and of $(\psi, \varepsilon)$-approximate quadratic *-derivations on $C^{*}$-algebras and show that every $(\psi$, $\varepsilon)$-approximate "-derivation is a "-derivation and that every $(\psi, \varepsilon)$-approximate quadratic "-derivation is a quadratic "-derivation. Thus, we extend the results of [21].

Definition 5.1 Suppose that $\mathcal{A}$ is $a^{*}$-normed algebra and $s \in\{1,-1\}$. Let $\delta: \mathcal{A} \rightarrow \mathcal{A}$ be a mapping for which there exist a mapping $\varepsilon: \mathcal{A} \rightarrow \mathcal{A}$ and a function $\psi: \mathcal{A} \times \mathcal{A} \rightarrow \mathbb{R}$ satisfying

$$
\lim _{n \rightarrow \infty} n^{-s} \psi\left(n^{s} a, b\right)=\lim _{n \rightarrow \infty} n^{-s} \psi\left(a, n^{s} b\right)=0(a, b \in \mathcal{A})
$$

such that

$$
\begin{aligned}
& \|a \delta(b)-\varepsilon(a) b\| \leq \psi(a, b) \\
& \|\varepsilon(a) c d-a(\delta(c) d-c \delta(d))\| \leq \psi(a, c d) \\
& \left\|a \delta(b)^{*}-\varepsilon(a) b^{*}\right\| \leq \psi(a, b)
\end{aligned}
$$

for all $a, b, c, d \in \mathcal{A}$. Then $\delta$ is called $a(\psi, \varepsilon)$-approximate *-derivation on $\mathcal{A}$.

Theorem 5.2 Let $\mathcal{A}$ be a $C^{*}$-algebra. Then any $(\psi, \varepsilon)$-approximate "-derivation $\delta$ on Ais $a^{*}$-derivation.

Proof. We assume that (5.1) holds. Let $a, b \in \mathcal{A}$ and $\lambda \in \mathbb{C}$. We have

$$
\begin{aligned}
\|b(\delta(\lambda a)-\lambda \delta(a))\| & \leq n^{-s}\left\|n^{s} b \delta(\lambda a)-\lambda n^{s} b \delta(a)\right\| \\
& \leq n^{-s}\left\|n^{s} b \delta(\lambda a)-\varepsilon\left(n^{s} b\right) \lambda a\right\|+n^{-s}\left\|\varepsilon\left(n^{s} b\right) \lambda a-\lambda n^{s} b \delta(a)\right\| \\
& \leq n^{-s} \psi\left(n^{s} b, \lambda a\right)+n^{-s}|\lambda| \psi\left(n^{s} b, a\right),
\end{aligned}
$$

which tends to zero as $n \rightarrow \infty$, and so $b(\delta(\lambda a)-\lambda \delta(a))=0$ for all $b \in \mathcal{A}$. Let $\left\{e_{i}\right\}_{i \in I}$ be an approximate unit of $\mathcal{A}$. If we replace $b$ with $\left\{e_{i}\right\}$, then we have

$$
\left\|e_{i}(\delta(\lambda a)-\lambda \delta(a))\right\|=0
$$

for all $i \in I$. So we conclude that $\delta(\lambda a)=\lambda \delta(a)$ for all $a \in \mathcal{A}$ and $\lambda \in \mathbb{C}$.

The additivity of $\delta$ follows from

$$
\begin{aligned}
& \|c(\delta(a+b)-\delta(a)-\delta(b))\| \\
& \left.\left.\quad \leq n^{-s}\left\|n^{s} c \delta(a+b)-\varepsilon\left(n^{s} c\right)(a+b)\right\|+n^{-s} \| n^{s} c \delta(a)-\varepsilon\left(n^{s} c\right) a\right)\left\|+n^{-s}\right\| n^{s} c \delta(b)-\varepsilon\left(n^{s} c\right) b\right) \\
& \quad \leq n^{-s} \psi\left(n^{s} c, a+b\right)+n^{-s} \psi\left(n^{s} c, a\right)+n^{-s} \psi\left(n^{s} c, b\right) .
\end{aligned}
$$

By the same process, using the approximate unit of $\mathcal{A}$, we have that $\delta(a+b)-\delta(a)$ $-\delta(b)$ for all $a, b \in \mathcal{A}$.

The following computation

$$
\begin{aligned}
\| & z(\delta(a b)-\delta(a) b-a \delta(b)) \| \\
& \leq n^{-s}\left\|n^{s} z \delta(a b)-\varepsilon\left(n^{s} z\right)(a b)\right\|+n^{-s}\left\|\varepsilon\left(n^{s} z\right) a b-n^{s} z(\delta(a) b+a \delta(b))\right\| \\
& \leq n^{-s} \psi\left(n^{s} z, a b\right)+n^{-s} \psi\left(n^{s} z, a b\right)
\end{aligned}
$$

yields that $\delta(a b)=\delta(a) b+a \delta(b)$ for all $a, b \in \mathcal{A}$. 
Finally, on the involution, we have that

$$
\begin{aligned}
\left\|z\left(\delta\left(a^{*}\right)-\delta(a)^{*}\right)\right\| \leq & n^{-s}\left\|n^{s} z \delta\left(a^{*}\right)-\varepsilon\left(n^{s} z\right) a^{*}\right\| \\
& +n^{-s}\left\|\varepsilon\left(n^{s} z\right) a^{*}-n^{s} z \delta(a)^{*}\right\| \\
\leq & n^{-s} \psi\left(n^{-s} z, a^{*}\right)+n^{-s} \psi\left(n^{s} z, a\right) .
\end{aligned}
$$

Thus, $\delta(a)^{*}=\delta(a) *$ for all $a \in \mathcal{A}$. $\square$

Therefore, $\delta$ is a "-derivation on $\mathcal{A}$.

Corollary 5.3 Suppose that $\mathcal{A}$ is a $C^{*}$-algebra and that $\delta: \mathcal{A} \rightarrow \mathcal{A}$ is a mapping for which there exist nonnegative real numbers $\alpha, \beta$ and positive real numbers $p_{1}, p_{2}, q_{1}$, $q_{2}$ with $p_{1}, p_{2}, q_{1}, q_{2}<1$ such that

$$
\begin{aligned}
& \|a \delta(b)-\varepsilon(a) b\| \leq \alpha\left(\|a\|^{p_{1}}+\|b\|^{p_{2}}\right)+\beta\|a\|^{q_{1}}\|b\|^{q_{2},} \\
& \|\varepsilon(a) c d-a(\delta(c) d-c \delta(d))\| \leq \alpha\left(\|a\|^{p_{1}}+\|c d\|^{p_{2}}\right)+\beta\|a\|^{q_{1}}\|c d\|^{q_{2}}, \\
& \left\|a \delta(b)^{*}-\varepsilon(a) b^{*}\right\| \leq \alpha\left(\|a\|^{p_{1}}+\|b\|^{p_{2}}\right)+\beta\|a\|^{q_{1}}\|b\|^{q_{2}}
\end{aligned}
$$

for all $a, b, c, d \in \mathcal{A}$. Then $\delta$ is a "-derivation of $\mathcal{A}$.

Next, we prove the superstability of quadratic "-derivations on $C^{*}$-algebras.

Definition 5.4 Suppose that $\mathcal{A}$ is $a$ "-normed algebra and $s \in\{-1,1\}$. Let $\delta: \mathcal{A} \rightarrow \mathcal{A}$ be a mapping for which there exist a function $\psi: \mathcal{A} \times \mathcal{A} \rightarrow[0, \infty)$ and a mapping $\lim _{n \rightarrow \infty} n^{-2 s} \psi\left(n^{s} a, b\right)=\lim _{n \rightarrow \infty} n^{-2 s} \psi\left(a, n^{s} b\right)=0(a, b \in \mathcal{A})_{\text {satisfying }}$

$$
\lim _{n \rightarrow \infty} n^{-2 s} \psi\left(n^{s} a, b\right)=\lim _{n \rightarrow \infty} n^{-2 s} \psi\left(a, n^{s} b\right)=0(a, b \in \mathcal{A})
$$

such that

$$
\begin{aligned}
& \left\|a^{2} \delta(b)-\varepsilon(a) b^{2}\right\| \leq \psi(a, b) \\
& \left\|\varepsilon(a)(c d)^{2}-a^{2}\left(\delta(c) d^{2}-c^{2} \delta(d)\right)\right\| \leq \psi(a, c d) \\
& \left\|a^{2} \delta\left(b^{*}\right)-\varepsilon(a)\left(b^{2}\right)^{*}\right\| \leq \psi(a, b)
\end{aligned}
$$

for all a,b,c,d $\in A$. Then $\delta$ is called a $(\psi, \varepsilon)$-approximate quadratic *-derivation on $\mathcal{A}$. Theorem 5.5 Suppose that $\mathcal{A}$ is a $C^{*}$-algebra and $s \in\{-1,1\}$. Let $\delta: \mathcal{A} \rightarrow \mathcal{A}$ be a $(\psi$, e)-approximate quadratic "-derivation on $\mathcal{A}$. Then $\delta$ is a quadratic "-derivation on $\mathcal{A}$.

Proof. We assume that (5.2) holds. We first show that $\delta$ is quadratic homogeneous. To do this, pick $\lambda \in \mathbb{C}$ and $a, b \in \mathcal{A}$. Then, we have

$$
\begin{aligned}
\left\|b^{2}\left(\delta(\lambda a)-\lambda^{2} \delta(a)\right)\right\| & =n^{-2 s}\left\|n^{2 s} b^{2} \delta(\lambda a)-\lambda^{2} n^{2 s} b^{2} \delta(a)\right\| \\
& \leq n^{-2 s}\left\|n^{2 s} b^{2} \delta(\lambda a)-\varepsilon\left(n^{s} b\right)(\lambda a)^{2}\right\|+n^{-2 s}\left\|\lambda^{2} \varepsilon\left(n^{s} b\right) a^{2}-\lambda^{2} n^{2 s} b^{2} \delta(a)\right\| \\
& \leq n^{-2 s} \psi\left(n^{s} b, \lambda a\right)+n^{-2 s}|\lambda|^{2} \psi\left(n^{s} b, a\right) .
\end{aligned}
$$

So

$$
\left\|b^{2}\left(\delta(\lambda a)-\lambda^{2} \delta(a)\right)\right\| \leq n^{-2 s} \psi\left(n^{s} b, \lambda a\right)+|\lambda|^{2} n^{-2 s} \psi\left(n^{s} b, a\right),
$$

which tends to 0 as $n \rightarrow \infty$. Let $\left\{e_{i}\right\}_{i \in I}$ be an approximate unit of $\mathcal{A}$. Then, $\left\{f\left(e_{i}\right) \mid i \in I\right\}$ is also an approximate unit of $\mathcal{A}$ for every polynomial $f$. Considering $e_{i}$ instead of $b$ in the above inequality, we conclude that $\delta(\lambda a)=\lambda^{2} \delta(a)$ for all $\lambda \in \mathbb{C}$. 
The quadraticity of $\delta$ follows from

$$
\begin{aligned}
\| & d^{2}(\delta(a+b)+\delta(a-b)-2 \delta(a)-2 \delta(b)) \| \\
= & n^{-2 s}\left\|n^{2 s} d^{2} \delta(a+b)+n^{2 s} d^{2} \delta(a-b)-2 n^{2 s} d^{2} \delta(a)-2 n^{2 s} d^{2} \delta(b)\right\| \\
\leq & n^{-2 s}\left[\left\|n^{2 s} d^{2} \delta(a+b)-\varepsilon\left(n^{s} d\right)(a+b)^{2}\right\|\right. \\
& +n^{-2 s}\left\|n^{2 s} d^{2} \delta(a-b)-\varepsilon\left(n^{s} d\right)(a-b)^{2}\right\| \\
& \left.+2 n^{-2 s}\left\|\delta\left(n^{s} d\right) a^{2}-n^{2 s} d^{2} \delta(a)\right\|+2 n^{-2 s}\left\|\delta\left(n^{s} d\right) b^{2}-n^{2 s} d^{2} \delta(b)\right\|\right] \\
\leq & n^{-2 s}\left[\psi\left(n^{s} d, a+b\right)+\psi\left(n^{s} d, a-b\right)+2 \psi\left(a, n^{s} d\right)+2 \psi\left(b, n^{s} d\right)\right]
\end{aligned}
$$

for all $a, b, d \in \mathcal{A}$. Thus, we have $\delta(a+b)+\delta(a-b)-2 \delta(a)-2 \delta(b)=0$ for all

$$
\begin{aligned}
\| d^{2}\left(\delta(a b)-\left(\delta(a) b^{2}+a^{2} \delta(b)\right) \|=\right. & n^{-2 s}\left\|n^{2 s} d^{2}\left(\delta(a b)-\delta(a) b^{2}-a^{2} \delta(b)\right)\right\| \\
\leq & n^{-2 s}\left[\left\|n^{2 s} d^{2} \delta(a b)-\varepsilon\left(n^{s} d\right)(a b)^{2}\right\|\right. \\
& \left.+n^{-2 s}\left\|\varepsilon\left(n^{s} d\right)(a b)^{2}-n^{2 s} d^{2} \delta(a) b^{2}+n^{2 s} d^{2} a^{2} \delta(b)\right\|\right] \\
\leq & n^{-2 s}\left[\psi\left(n^{s} d, a b\right)+\psi\left(n^{s} d, a b\right)\right] \\
\| d^{2}\left(\delta(a b)-\left(\delta(a) b^{2}+a^{2} \delta(b)\right) \|=\right. & n^{-2 s}\left\|n^{2 s} d^{2}\left(\delta(a b)-\delta(a) b^{2}-a^{2} \delta(b)\right)\right\| \\
\leq & n^{-2 s}\left[\left\|n^{2 s} d^{2} \delta(a b)-\varepsilon\left(n^{s} d\right)(a b)^{2}\right\|\right. \\
& \left.+n^{-2 s}\left\|\varepsilon\left(n^{s} d\right)(a b)^{2}-n^{2 s} d^{2} \delta(a) b^{2}+n^{2 s} d^{2} a^{2} \delta(b)\right\|\right] \\
\leq & n^{-2 s}\left[\psi\left(n^{s} d, a b\right)+\psi\left(n^{s} d, a b\right)\right]
\end{aligned}
$$

for all $a, b, d \in \mathcal{A}$. So $\delta(a b)=\delta(a) b^{2}+a^{2} \delta(b)$.

The rest of the proof is similar to the proof of Theorem 5.2.

Therefore, $\delta$ is a quadratic "-derivation on $\mathcal{A}$.

Corollary 5.6 Suppose that $\mathcal{A}$ is a $C^{*}$-algebra and that $\delta: \mathcal{A} \rightarrow \mathcal{A}$ is a mapping for which there exist a nonnegative real number $\alpha$ and a positive real number $p$ with $p<2$ such that

$$
\begin{aligned}
& \left\|a^{2} \delta(b)-\delta(a) b^{2}\right\| \leq \alpha\|a\|^{p}\|b\|^{p}, \\
& \left\|\varepsilon(a)(c d)^{2}-a^{2}\left(\delta(c) d^{2}-c^{2} \delta(d)\right)\right\| \leq \alpha\|a\|^{p}\|c d\|^{p}, \\
& \left\|a^{2} \delta\left(b^{*}\right)-\varepsilon(a)\left(b^{2}\right)^{*}\right\| \leq \alpha\|a\|^{p}\|b\|^{p}
\end{aligned}
$$

for all $a, b, c, d \in A$. Then $\delta$ is a quadratic "-derivation on $\mathcal{A}$.

\section{Acknowledgements}

The first author and the second author were supported by Basic Science Research Program through the National Research Foundation of Korea funded by the Ministry of Education, Science and Technology (NRF-2010-0013211) and (NRF-2009-0070788), respectively.

\section{Author details}

${ }^{1}$ Department of Mathematics, University of Ulsan, Ulsan 680-749, Korea ${ }^{2}$ Department of Mathematics, Research Institute for Natural Sciences, Hanyang University, Seoul 133-791, Korea

\section{Authors' contributions}

All authors conceived of the study, participated in its design and coordination, drafted the manuscript, participated in the sequence alignment, and read and approved the final manuscript.

\section{Competing interests}

The authors declare that they have no competing interests.

Received: 17 March 2011 Accepted: 14 September 2011 Published: 14 September 2011

References

1. Bratteli, O: Derivation, dissipation and group actions on $C^{*}$-algebras. In Lecture Notes in Mathematics, vol. 1229,Springer, Berlin (1986) 
2. Bratteli, O, Goodman, FM, Jørgensen, PET: Unbouded derivations tangential to compact groups of automorphisms II. J Funct Anal. 61, 247-289 (1985). doi:10.1016/0022-1236(85)90022-9

3. Lee, S, Jang, S: Unbounded derivations on compact actions of $C^{*}$-algebras. Commun Korean Math Soc. 5, 79-86 (1990)

4. Baker, J: The stability of the cosine equation. Proc Am Math Soc. 80, 242-246 (1979)

5. Ulam, SM: Problems in Modern Mathematics, chapt. VI, Science edn. Wiley, New York (1940)

6. Hyers, DH: On the stability of the linear functional equation. Proc Nat Acad Sci USA. 27, $222-224$ (1941). doi:10.1073/ pnas.27.4.222

7. Rassias, ThM: On the stability of the linear mapping in Banach spaces. Proc Am Math Soc. 72, 297-300 (1978). doi:10.1090/S0002-9939-1978-0507327-1

8. Aczl, J, Dhombres, J: Functional Equations in Several Variables. Cambridge University Press, Cambridge (1989)

9. Chou, C-Y, Tzeng, J-H: On approximate isomorphisms between Banach *-algebras or $C^{*}$-algebras. Taiwan J Math. 10 219-231 (2006)

10. Czerwik, S: On the stability of the quadratic mapping in normed spaces. Abh Math Sem Univ Hambg. 62, 59-64 (1992). doi:10.1007/BF02941618

11. Gajda, Z: On stability of additive mappings. Intern J Math Math Sci. 14, 431-434 (1991). doi:10.1155/S016117129100056X

12. Hyers, DH, Isac, G, Rassias, ThM: Stability of Functional Equations in Several Variables. Birkhäuser, Basel (1998)

13. Jun, K, Kim, H: Approximate derivations mapping into the radicals of Banach algebras. Taiwan J Math. 11, 277-288 (2007)

14. Kannappan, PI: Quadratic functional equation and inner product spaces. Results Math. 27, 368-372 (1995)

15. Skof, F: Propriet locali e approssimazione di operatori. Rend Sem Mat Fis Milano. 53, 113-129 (1983). doi:10.1007/ BF02924890

16. Lee, J, An, J, Park, C: On the stability of qudratic functional equations. Abstr Appl Anal 2008, 8 (2008). (Art ID 628178)

17. Gharetapeh, SK, Gordji, ME, Ghaemi, MB, Rashidi, E: Ternary Jordan homomorphisms in C*-ternary algebras. J Nonlinear Sci Appl. 4, 1-10 (2011)

18. Park, C, Boo, D: Isomorphisms and generalized derivations in proper CQ*-algebras. J Nonlinear Sci Appl. 4, 19-36 (2011)

19. Javadian, A, Gordji, ME, Savadkouhi, MB: Approximately partial ternary quadratic derivations on Banach ternary algebras. J Nonlinear Sci Appl. 4, 60-69 (2011)

20. Cholewa, PW: Remarks on the stability of functional equations. Aequ Math. 27, 76-86 (1984). doi:10.1007/BF02192660

21. Moslehian, MS, Rahbarnia, F, Sahoo, PK: Approximate double centralizers are exact double centralizers. Bol Soc Mat Mex. $13,111-122(2007)$

doi:10.1186/1029-242X-2011-55

Cite this article as: Jang and Park: Approximate *-derivations and approximate quadratic *-derivations on $C^{*}$ algebras. Journal of Inequalities and Applications 2011 2011:55.

\section{Submit your manuscript to a SpringerOpen ${ }^{\circ}$ journal and benefit from:}

- Convenient online submission

- Rigorous peer review

- Immediate publication on acceptance

- Open access: articles freely available online

- High visibility within the field

- Retaining the copyright to your article

Submit your next manuscript at $\gg$ springeropen.com 